\title{
EPOS 3 and Air Showers
}

\author{
Tanguy Pierog ${ }^{1, *}$, Benjamin Guiot ${ }^{2}$, Iurii Karpenko ${ }^{3}$, Gabriel Sophys ${ }^{3}$, Maria Stefaniak ${ }^{4}$, and Klaus Werner $^{3}$ \\ ${ }^{1}$ Karlsruhe Institute of Technology, Institut für Kernphysik, Karlsruhe Germany \\ ${ }^{2}$ Universidad Técnica Federico Santa Maria - Departamento de Fisica - Casilla 110-V, Valparaíso, Chile \\ ${ }^{3}$ SUBATECH, University of Nantes - IN2P3/CNRS- EMN, Nantes, France \\ ${ }^{4}$ Warsaw University of Technology, Warsaw, Poland
}

\begin{abstract}
With the recent results of large hybrid air-shower experiments, it is clear that the simulations of the hadronic interactions are not reliable enough to obtain a consistent description of the observations. Even the most recent models tuned after the first run of the LHC show significant discrepancy with air-shower data. Since then many more data have been collected at the LHC and at lower energies at the SPS which are not necessarily well described by these models. So before claiming any explanation involving new physics, it is necessary to have a model which can actually describe accelerator data in a very detailed way. That is the goal of EPOS 3, to understand both soft and hard particle production not only in light systems like proton-proton interactions but in heavy ions too. The latest results of the model will be presented and in particular the correlations between various observables which are very important to understand the real physical processes.
\end{abstract}

\section{Introduction}

Despite all the efforts done to take into account the first results of proton-proton collisions at LHC in hadronic interaction models used for air-shower simulations, the observed number of muons, their height of production or even the depth of shower maximum are still not reproduced consistently by the models [1]. Furthermore, the difference in model predictions introduce uncertainty in cosmic ray data analysis which is lower than in the past but still larger than experimental uncertainty in certain cases [2]. But before claiming for the need for "new physics", it is important to guarantee that all the standard physics of QCD is properly taken into account in these models. For that it is necessary to go beyond the simplest observables which are usually used to test the models and LHC experiments provided a large amount of complex data to analyse and understand, in particular thanks to the correlation between different observables.

Among the hadronic interaction models used for airshower analysis, only Epos [3-5] includes all the features needed to have a detailed description of the correlation between various observables [1]. Indeed the core-corona approach in this model which allows the production of a collective phase appears to be a key element to reproduce LHC data. Before LHC run, it was usually accepted that hydrodynamical phase expansion due to the formation of a quark-gluon plasma (QGP) for instance was possible only in central heavy ion (HI) collisions. Proton-nucleus (pA) collisions were then used as a reference to probe the effect of such collective behavior (final state effect) but with some nuclear effect at the initial state level, while proton-

*e-mail: tanguy.pierog@kit.edu proton (pp) interactions were free of any nuclear effect. With $\mathrm{LHC}$ run in $\mathrm{pp}, \mathrm{pPb}$ and $\mathrm{PbPb}$ mode, it is now possible to compare high multiplicity $\mathrm{pp}$ or $\mathrm{pPb}$ events with low multiplicity $\mathrm{PbPb}$ events (which correspond to the same number of particles measured at mid-rapidity) and surprisingly the very same phenomena are observed $[6,7]$ concerning the soft particle production.

One of the most striking features observed in all systems is the long-range two-particle correlations and the evolution of the particle flow as described in [8]. In [9] the authors demonstrate how these data from the CMS collaboration can be reproduced and explained using an approach combining standard perturbative calculations for initial conditions and hydrodynamical calculations for the final state interactions. This study is based on the Epos model version $3.2 \mathrm{x}$ in which a parametrized saturation scale is used. In this paper, we will present the difference between Epos LHC and Epos 3 and their consequences for air-shower physics. In particular, a new approach has been implemented to calculate the saturation scale in Epos which allows a better setting of the initial conditions to test the effect of collective hadronization. We will demonstrate that this effect, which was attributed to heavy ion collisions only, is very important to describe pp data in particular for strange particle production and that it is potentially more important for air showers than initially thought.

In Section 2 the basic principles of Epos 3 will be presented. In Section 3 a new way of calculating the saturation scale on an event-by-event basis will be introduced, and in Section 4 we will present the changes on collective hadronization. Section 5 is dedicated to changes at large rapidities which will have a direct impact on muon production. Finally, in Section 6, we will summarize the ex- 
pected impact of the new features of Epos 3 on air-shower simulations.

\section{EPOS 3}

In order to make a hydrodynamical evolution calculation, proper initial conditions are needed. In our approach, the Epos 3 [10] model is used to determine the energy-density tensor and flavor content of the thermalized matter and to solve the differential equations of the hydrodynamical calculation.

Epos 3 is a minimum bias Monte Carlo hadronic generator used for heavy ion interactions. It is the last generation of a long development of the Epos model [3-5]. It is the only hadronic model which has a consistent treatment of cross-section calculation and particle production taking into account energy conservation in the former and in the later and thanks to the parton-based Gribov-Regge theory [11]. In this approach, the basic ingredient is the purely imaginary amplitude of a single Pomeron exchange which is the sum of a (parametrized) soft contribution (Regge-like after a Fourier transformation from the $t$ space to the impact parameter $b$ space) $G_{0}(\hat{s}, b)=\alpha_{0}(b) \hat{s}^{\beta_{0}}$ and a semi-hard contribution based on the convolution of a soft pre-evolution, a DGLAP [12] based hard evolution and a standard leading order QCD $2 \rightarrow 2$ cross-section (mini-jet). The latter (called $\hat{G}$ ) needs complex calculations but can be fitted to a simple Regge-like term: $G_{1}(\hat{s}, b)=\alpha_{1}(b) \hat{s}^{\beta_{1}}$. Here $\hat{s}=s x^{+} x^{-}$is the fraction of the center-of-mass energy squared (mass) carried by the Pomeron and $b$ the impact parameter of the nucleon-nucleon collision. Details can be found in [11].

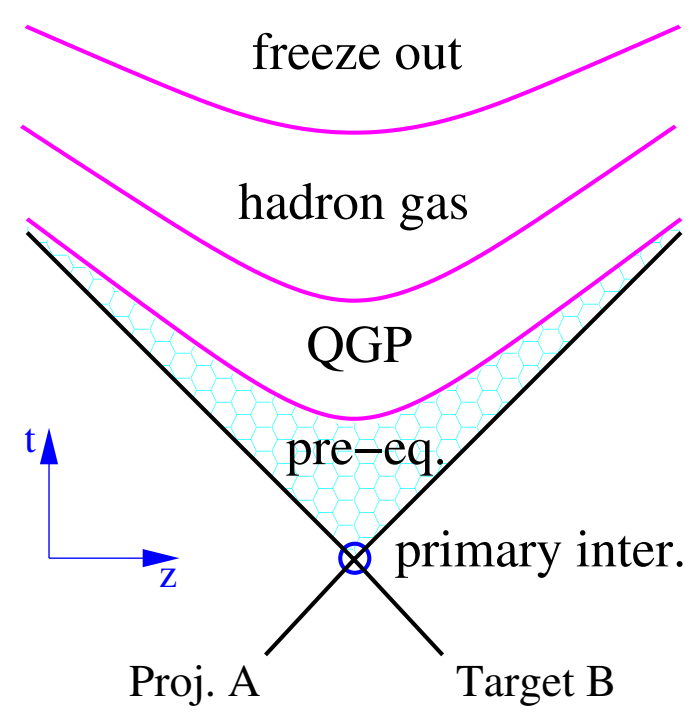

Figure 1. General space-time evolution of particles in hadronic collisions. The combination of QGP and hadron gas apply to the "core" only and the combination of both is called collective hadronization.

Both cross-sections and particle production are based on the total amplitude $G=\sum_{i} G_{i}$ via a complex Markov-
Chain Monte Carlo. The particle production has two main components: the strings composed from the Pomerons which cover the mid-rapidity part and the remnants which carry the remaining energy and quarks and cover mostly the fragmentation region. The strings, two per Pomeron, include the initial state radiation and final state radiation and the soft contribution from the non-perturbative preevolution (below the fixed scale $Q_{0}^{2}$ ). At high energy many Pomerons can happen in parallel for each event in multiple parton interaction (MPI). A remnant can be as simple as a resonance or a string elongated along the beam axis if its mass is too high, and is treated in the same way for both diffractive and non-diffractive events.

The string fragments are then used to compute the energy-density tensor on an event-by-event basis. If the energy density is higher than some threshold, string segments are merged locally into the so-called "core" to form the initial conditions of a system of a hydrodynamical differential equation with an equation of state based on lattice QCD. Details can be found in [4].

In Fig. 1 a simplified schematic view of the spacetime evolution of the "core" part in Epos 3 is represented. The details of the collective hadronization phase between the primary interaction, in which the initial conditions are created, and the freeze out, after which particles travel without reinteraction, change on an event-by-event basis. The energy, impact parameter, number of multiple scatterings and geometry all change the initial conditions and the possible existence and evolution of the "core" but the same rules apply whatever energy and system is considered (from pp to heavy ions). In practice there is no real phase boundary between the QGP and the hadron gas in this collective hadronization phase.

\section{Saturation scale}

To correct the limitation observed in Epos LHC [3] for high transverse momentum (pt) particles, in particular in proton-nucleus ( $\mathrm{pA})$, a new saturation scale has been introduced which can be different for each Pomeron. In Epos LHC and previous versions, non-linear effects due to Pomeron-Pomeron interactions were treated by a simple correction on the $\beta_{i}$ exponent of the $G_{i}$ contributions of the Pomeron amplitude [5]. But this approach was changing both the soft (multiplicity) and the hard component (high pt) in the same way. Since strong nuclear effects are needed to reproduce both the cross-section and the multiplicity of $\mathrm{pA}$ interactions leading to a strong correction on $\beta$, a strong suppression of high pt particles was observed in Epos simulations.

Instead of applying the correction on $\beta$ to the real Pomeron amplitude $\hat{G}$, it is possible to change $\hat{G}$ itself to reproduce the modified $G$ (called $\tilde{G}$ ) simply by changing the scale at which the perturbative calculation is done: $Q_{0}^{2} . \quad Q_{0}^{2}$ is replaced by $Q_{0}^{2}\left(x^{+}, x^{-}, s, b\right)$ to calculate each Pomeron amplitude. It is in fact possible to calculate $Q_{0}^{2}$ by the generation of Pomerons using the effective $\tilde{G}$ which reproduces the cross-section and the multiplicity observed in the data. Since we want to recover the binary scaling at 
high pt we can use

$$
\tilde{G}\left(x^{+}, x^{-}, s, b\right) \propto N_{P} \hat{G}\left(x^{+}, x^{-}, s, b, Q_{0}^{2}\left(x^{+}, x^{-}, s, b\right)\right)
$$

to compute $Q_{0}^{2}$ Pomeron-by-Pomeron. $N_{\boldsymbol{P}}$ is the actual number of Pomerons connected to each nucleon of the considered pair (so taking into account the connection with other nucleons outside this pair in case of nuclear collisions). Integrating over all minimum bias events, the factor $N_{\boldsymbol{P}}$ gives $\left\langle N_{\boldsymbol{P}}\right\rangle_{\text {pair }}$ in case of pp but $\left\langle N_{\boldsymbol{P}}\right\rangle_{\text {pair }} \times\left\langle N_{b i n}\right\rangle$ for $\mathrm{pA}$, where $\left\langle N_{b i n}\right\rangle$ is the number of binary collisions, leading to a perfect binary scaling (beyond $Q_{s}^{2}$ ).

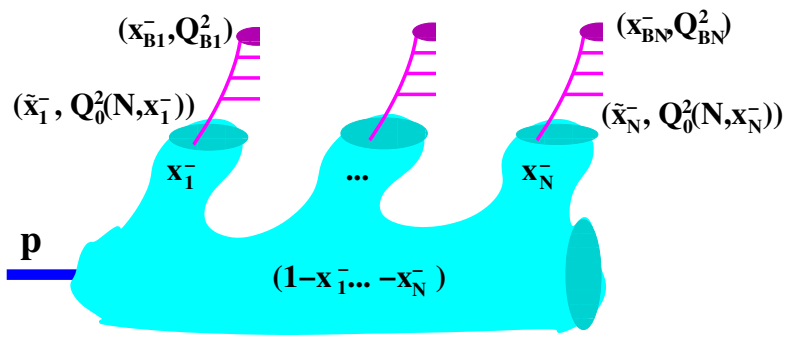

Figure 2. Schematic view of the Pomeron generation on the target side of a pp interaction. In brackets are the momentum fraction and virtuality of the parton at the beginning $\left(\tilde{x}, Q_{0}^{2}\right)$ and at the end $\left(x_{\mathrm{B}}, Q_{\mathrm{B}}^{2}\right)$ of the DGLAP evolution.

Epos 3, in fact, generates some kind of generalized parton distribution function which depends on the MPI configuration. The energy sharing creates correlations in the generated momentum fraction, and the virtuality at which the perturbative calculation is done depends on both the multiple scattering and the momentum fraction as represented in Fig. 2.

To illustrate the fact that a change of $Q_{0}^{2}$ is a way to reduce the soft parton production without changing the hard ones (above $Q_{0}^{2}$ ), the deviation between Epos 3.223 calculation of the transverse momentum distribution of the jets rescaled by the number of binary collisions and a pure pQCD calculation using Cteq6 [13] parton distribution functions can be observed in Fig. 3 for central pPb collisions at $5 \mathrm{TeV}$. The high pt part is in perfect agreement while a strong suppression is observed at low pt.

The same scheme to compute $Q_{0}^{2}$ is of course used for pp interactions, and with enough MPI it solves the problem observed in Epos LHC [3] with high pt leading particles in the underlying event (UE) activity. The measurement of the UE activity was initiated by the CDF collaboration more than ten years ago [14] to understand multiple parton interactions and test the capability of the event generators to reproduce real events. This analysis has been generalized to more recent collider data including the main LHC experiments ATLAS [15] and CMS [16]. In fact, the effect of using $N_{\boldsymbol{P}}$ in Eq. 1 instead of a direct equality is very important and can be observed in Fig. 4 by the difference between the dashed line ( $N_{\boldsymbol{P}}$ not taken into account) and the dash-dotted line ( $N_{\boldsymbol{P}}$ taken into account). In both cases, MPI is the same, and the inclusive jet cross-section is correct, but using $N_{\boldsymbol{P}}$ in Eq. 1 means to take into account MPI in the saturation scale too and is necessary to

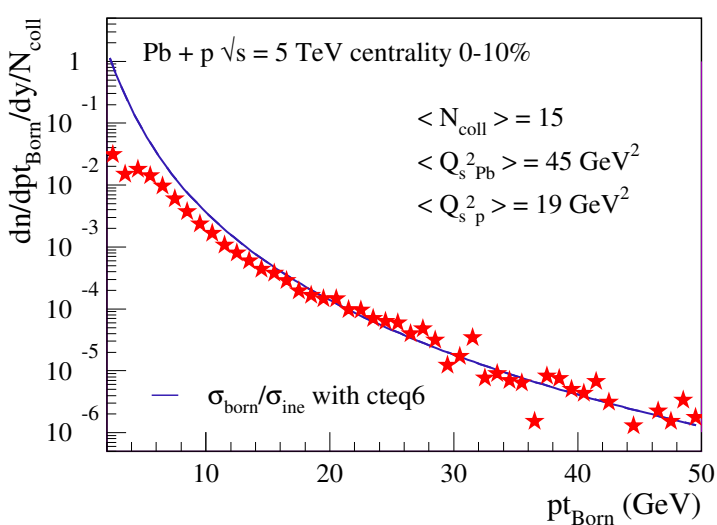

Figure 3. Born parton pt distribution normalized by the Glauber number of binary collisions from $\mathrm{pPb}$ collisions at $5.02 \mathrm{TeV}$ for the $0-10 \%$ centrality bin. Epos 3.223 simulations are shown with stars and compared to the normalized inclusive cross-section (full line).

reproduce the data (for all charged particles for instance). The improvement of the pQCD part of the model allows, in addition, the production of heavy quarks such as charm and beauty and the corresponding hadrons.

\section{Collective hadronization}

\subsection{Light systems}

Another UE analysis is of particular interest when focusing on the effect of collective hadronization since it is based on the strange particle production [17]. Indeed the strange particles and in particular strange baryons are more sensitive to hydrodynamical effects since statistical hadronization which occurs at the surface of the fluid leads to an increase of strangeness production compared to usual string fragmentation and the transverse momentum flow due to the larger fluid evolution for heavier particles.

In Fig. 4 we can compare the $\Lambda$ production in underlying event activity in Epos 3 with (full line) or without (dash-dotted line) collective hadronization. Without this effect, the strange baryon production is clearly underestimated as observed for other Monte Carlo generators in [17] while the data can be nicely reproduced if the formation of a core which hadronizes statistically is taken into account. We can conclude that underlying event activity measurements, and in particular, whose related to strange particles, can be used to test collective hadronization effects in proton-proton collisions and are necessary to reproduce even the pp data.

\subsection{Heavy systems}

For many years, it is well established that collective hadronization is a must to reproduce data taken with heavy ions (HI) such as gold or lead. Thanks to the measurement of various observables as a function of the particle multiplicity done at $\mathrm{LHC}$, it is possible to combine the different 


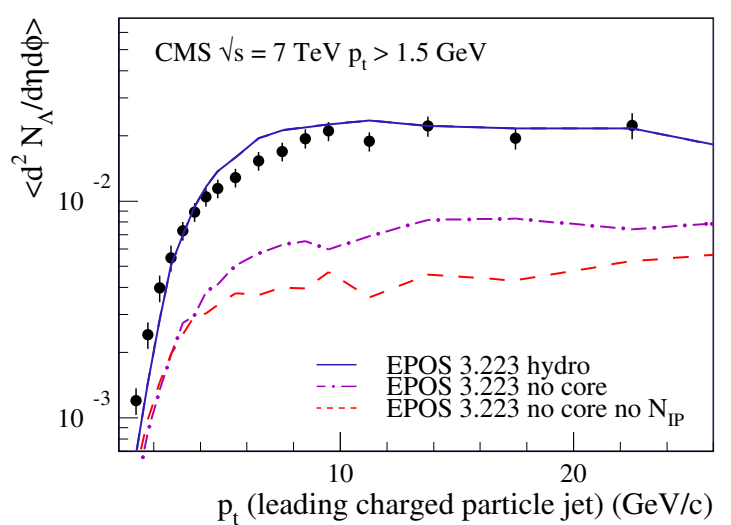

Figure 4. CMS measurement [17] of the number of $\Lambda$ particles with $p_{\mathrm{t}} \geq 1.5 \mathrm{GeV} / \mathrm{c}$ produced in the underlying event, i.e. a region of $60^{\circ} \leq|\Delta \phi| \leq 120^{\circ}$ around the leading particle as a function of the transverse momentum of the leading charged-particle jet. Epos 3.223 simulations are shown (see text for details).

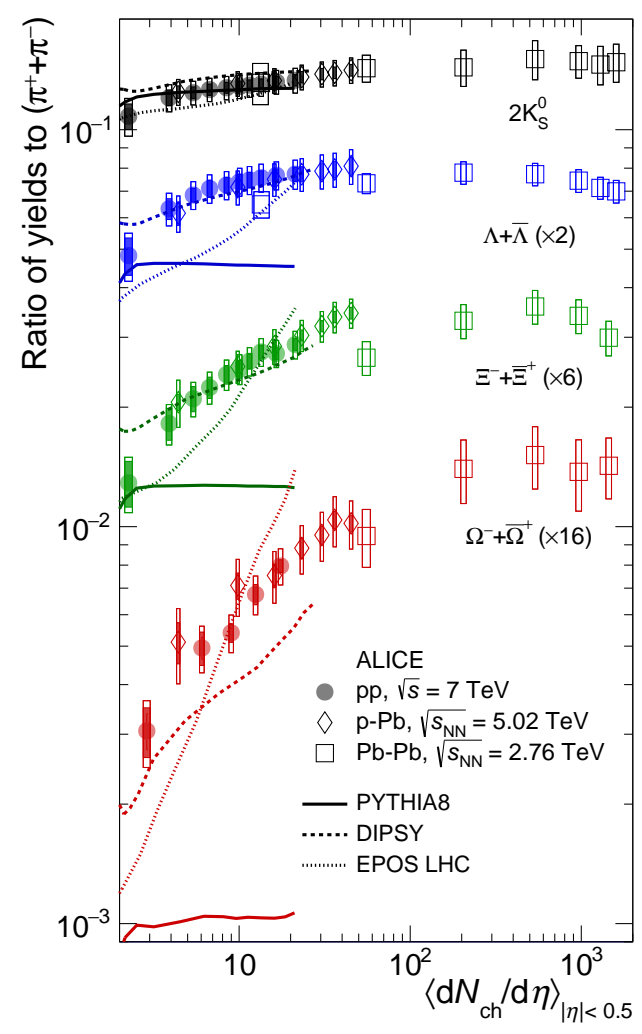

Figure 5. pt-integrated yield ratios to pions $\left(\pi^{+}+\pi^{-}\right)$as a function of $\left\langle\mathrm{d} N_{\mathrm{ch}} / \mathrm{d} \eta\right\rangle$ measured in $|y|<0.5$. The error bars show the statistical uncertainty, whereas the empty and dark-shaded boxes show the total systematic uncertainty and the contribution uncorrelated across multiplicity bins, respectively. The values are compared to calculations from MC models $[3,18,19]$ and to results obtained in pp and $\mathrm{pPb}$ collisions at the LHC [20-22]. For $\mathrm{PbPb}$ results, the ratio $2 \Lambda /\left(\pi^{+}+\pi^{-}\right)$is shown. The indicated uncertainties all represent standard deviations. Picture and caption are taken from [6]. systems such as $\mathrm{pp}, \mathrm{pPb}$ and $\mathrm{PbPb}$ in a single plot and see whether different behaviors are observed. As shown in Fig. 5 from [6], no particular difference has been seen for the various systems and there is a smooth transition from low to high multiplicity in strangeness production for instance. Furthermore, this transition does not occur only at a very high multiplicity reachable in $\mathrm{PbPb}$ collisions, but already starts at a quite low multiplicity with a sizable effect at the average multiplicity of pp interactions $\left(\left\langle\mathrm{d} N_{\mathrm{ch}} / \mathrm{d} \eta\right\rangle\right.$ about 6).

As seen in Fig. 5, Epos LHC was overestimating the multiplicity of this transition. The core with collective hadronization is produced too late compared to what is observed at LHC now (and the effect was overestimated to compensate and reproduce the average correctly). An example of what can be done with Epos 3 is visible in Fig. 6. In that case, the evolution of the number of $\Omega$ baryons as a function of the multiplicity is well reproduced both in shape and amplitude. We can see that the yield ratios of the different contributions (core (dotted line) or corona (dashdotted line)) do not depend neither on the system type nor on the multiplicity. The change of the ratio as a function of the multiplicity is only due to the evolution of the different fractions of particles produced by the core and the corona. On top of this, the effect of the hadron gas applies only in high multiplicity $\mathrm{PbPb}$ events (the difference between the dashed and the full line).

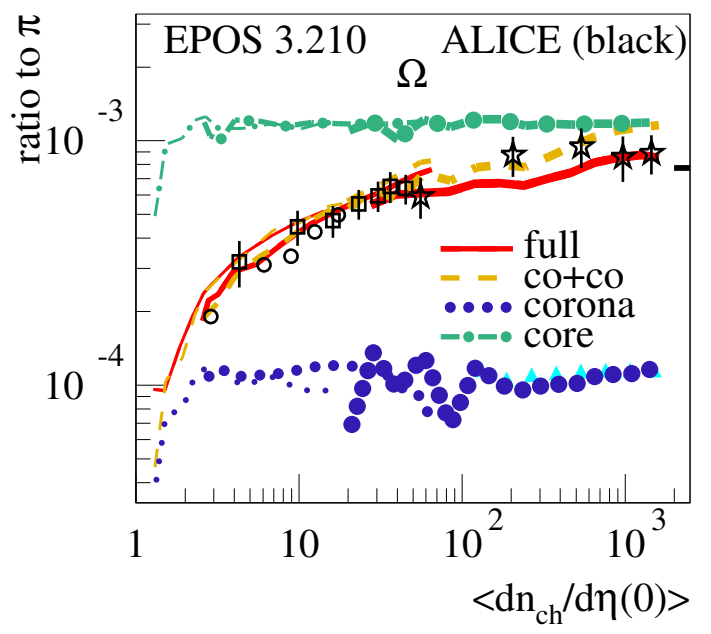

Figure 6. Particle to pion ratio for the $\Omega$ baryon versus multiplicity at mid-rapidity, for different contributions (core (dash-dotted), corona (dotted), core+corona (dashed) and all (core+corona+hadronic gas) (full)) from the EPOS simulations, for different systems (pp (thin), $\mathrm{pPb}$ (normal), $\mathrm{PbPb}$ (bold)). We also plot ALICE data from [6].

\section{Forward baryons and resonances}

One particular point of Epos LHC is that it produces a large amount of forward baryon leading to a larger but deeper muon production which appears to be in contradiction with some measurements of the Pierre Auger Observatory [23]. Epos predictions was based on old data of $\pi^{+}$ 
interactions only. With more recent data from NA49 [24] and NA61 [25] and both $\pi^{+}$and $\pi^{-}$interactions, the models can be better constrained and it appears that the situation is not symmetric as anticipated. In Fig. 7, we can observe that in fact only the forward proton production is enhanced more than the others. The mechanism is then not a larger pair production as done in EPos LHC but most probably a strong baryon stopping (which means an acceleration of the target nucleon in that case). This effect will be corrected in Epos 3.

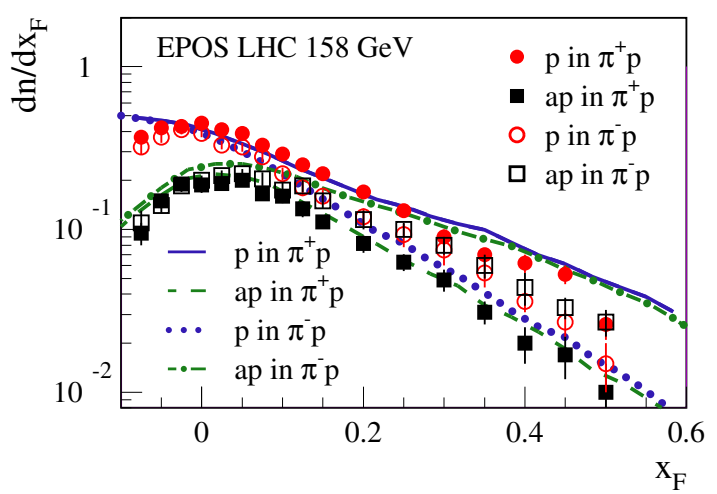

Figure 7. Number of protons and antiprotons produced in $\pi^{+}$ and $\pi^{-}$interactions with protons at $158 \mathrm{GeV}$ laboratory energy as a function of the Feynman momentum fraction $x_{\mathrm{F}}$. Data from NA49 [24] (points) are plotted together with Epos LHC simulations (lines).

Both NA61 $\rho^{0}$ measurements [26] and LHCf [27, 28] neutron measurements confirm a non-negligible pion exchange cross-section which was not properly implemented in Epos LHC. This processus will be added in Epos 3 too and change the forward production of nucleons and $\rho^{0}$ in case of pion interactions.

\section{Conclusion on air-shower physics}

Epos 3 will be a major evolution of Epos LHC motivated first of all by the need for a better description of high pt data at LHC both in light and heavy systems but with probably important consequences on air-shower physics. Since the new saturation scale described here will allow for a better description of hard probes, the perturbative QCD part of the Pomeron has been improved and will include the production of heavy quarks. It allows a very good test of the model at LHC which is very important for the prediction of high energy muons and neutrinos in atmospheric air showers.

The improvement of forward particle production thanks to the introduction of the pion exchange process and a better baryon stopping at low energy will have an impact on the muon production in particular in the last hadronic generation and then probably on the muons at large distance from the air-shower core. It will allow in addition to run Epos down to relatively low energy and reduce the role of the low hadronic interaction models which are more difficult to modify.
The better description of the collective hadronization and in particular the fact that the core is produced earlier than predicted by Epos LHC might have very important consequences for muon production in air showers. The effect of QGP was tested using Epos LHC and shown not to be significant. Indeed in this model, the QGP was produced only for very high multiplicity events and at midrapidity which are both rare and not so important for air-shower development. Other studies using a QGP or alternative hadronization as a possible new source of muons were all based on changes under extreme conditions too $[29,30]$ or with extreme consequences not observed at LHC [31]. As shown here, according to the most recent LHC results, the collective hadronization happens at a much lower multiplicity and as a consequence with effects at larger rapidities (lower particle densities than foreseen). In that case, much more particles coming from the hadronization of a QGP may play a significant role in the air-shower development. And as shown in [32], the energy fraction carried by the $\pi^{0}$ compared to the one carried by the other particles is smaller in such type of particle production. Thus a larger muon production is expected [33]. The production of QGP is increasing with energy (since the multiplicity increases) and as a consequence the number of produced muons should also increase faster with the primary energy as observed by the WHISP working group [34]. Since this effect will be implemented in Epos 3, we will see if this works quantitatively and not only qualitatively.

\section{Acknowledgments}

The authors are grateful to Carola Dobrigkeit for her careful reading and comments.

\section{References}

[1] T. Pierog, PoS ICRC2017, 1100 (2018)

[2] K.H. Kampert, M. Unger, Astropart.Phys. 35, 660 (2012), 1201.0018

[3] T. Pierog, I. Karpenko, J.M. Katzy, E. Yatsenko, K. Werner, Phys. Rev. C92, 034906 (2015), 1306.0121

[4] K. Werner, I. Karpenko, T. Pierog, M. Bleicher, K. Mikhailov, Phys. Rev. C82, 044904 (2010), 1004.0805

[5] K. Werner, F.M. Liu, T. Pierog, Phys. Rev. C74, 044902 (2006), hep-ph/0506232

[6] J. Adam et al. (ALICE), Nature Phys. (2017), 1606.07424

[7] J. Pan (ALICE), J. Phys. Conf. Ser. 832, 012044 (2017)

[8] V. Khachatryan et al. (CMS), Phys. Lett. B742, 200 (2015), 1409. 3392

[9] K. Werner, J. Phys. Conf. Ser. 636, 012006 (2015)

[10] K. Werner, B. Guiot, I. Karpenko, T. Pierog (2013), 1312.1233 
[11] H.J. Drescher, M. Hladik, S. Ostapchenko, T. Pierog, K. Werner, Phys. Rept. 350, 93 (2001), hep-ph/0007198

[12] G. Altarelli, G. Parisi, Nucl. Phys. B126, 298 (1977)

[13] J. Pumplin et al., JHEP 07, 012 (2002), hep-ph/0201195

[14] A.A. Affolder et al. (CDF), Phys. Rev. D65, 092002 (2002)

[15] G. Aad et al. (Atlas Collaboration), Phys.Rev. D83, 112001 (2011), 1012.0791

[16] S. Chatrchyan et al. (CMS), JHEP 09, 109 (2011), 1107.0330

[17] S. Chatrchyan et al. (CMS), Phys. Rev. D88, 052001 (2013), 1305.6016

[18] T. Sjöstrand, S. Mrenna, P.Z. Skands, Comput. Phys. Commun. 178, 852 (2008), 0710. 3820

[19] C. Bierlich, J.R. Christiansen, Phys. Rev. D92, 094010 (2015), 1507.02091

[20] B.B. Abelev et al. (ALICE), Phys. Lett. B728, 25 (2014), 1307.6796

[21] B.B. Abelev et al. (ALICE Collaboration), Phys.Lett. B728, 216 (2014), 1307. 5543

[22] J. Adam et al. (ALICE), Phys. Lett. B758, 389 (2016), 1512.07227

[23] A. Aab et al. (Pierre Auger), Phys. Rev. D90, 012012 (2014), [Erratum: Phys.
Rev.D92,no.1,019903(2015)], 1407. 5919

[24] G.I. Veres, Ph.D. thesis, Eötvös Loránd University, Budapest (2001)

[25] R.R. Prado (NA61/SHINE), PoS ICRC2017, 315 (2018), 1707.07902

[26] A. Aduszkiewicz et al. (NA61/SHINE), Eur. Phys. J. C77, 626 (2017), 1705.08206

[27] O. Adriani et al. (LHCf), Phys. Lett. B750, 360 (2015), 1503.03505

[28] O. Adriani et al. (LHCf), JHEP 11, 073 (2018), [JHEP18,073(2020)], 1808.09877

[29] D. LaHurd, C.E. Covault, JCAP 1811, 007 (2018), 1707.01563

[30] J.F. Soriano, L.A. Anchordoqui, T.C. Paul, T.J. Weiler, PoS ICRC2017, 342 (2018), 1811.07728

[31] J. Alvarez-Muniz, L. Cazon, R. Conceição, J.D. de Deus, C. Pajares, M. Pimenta (2012), 1209. 6474

[32] S. Baur, H. Dembinski, T. Pierog, R. Ulrich, K. Werner, To be published in Phys. Rev. D (2019)

[33] J. Matthews, Astropart. Phys. 22, 387 (2005)

[34] H. Dembinski, J. Arteaga-Velázquez, L. Cazon, R. Conceição, J. Gonzalez, Y. Itow, D. Ivanov, N. Kalmykov, I. Karpikov, T. Pierog et al. (EASMSU, KASCADE-Grande, IceCube, NEVODDECOR, Pierre Auger, SUGAR, Telescope Array, Yakutsk EAS), This proceedings (2019) 